InnOvaciOnes de NegOciOs 17(33): 86-97

(C) 2020 UANL, Impreso en México (ISSN: 2007-1191)

Recepción: 12 Diciembre 2019 Aceptación: 10 Enero 2019

\title{
Constitución legal y registro de las MiPymes de Tepexi de Rodríguez \\ (Legal constitution and registration of the MSMEs of Tepexi de Rodríguez)
}

\author{
Socorro Pacheco Pérez, Rosa Isela Escandón Rivera y Jesús Pérez Sánchez \\ Instituto Tecnológico Superior de Tepexi de Rodríguez \\ pachecopsocorro@gmail.com, iselaescandonrivera@gmail.com, \\ jesuaeternal@gmail.com
}

\begin{abstract}
One of the obligations of every merchant is the inscription of his business in the Public Registry of Commerce, this guarantees knowledge about the economic entities of the country and encourages formal commerce. In Tepexi de Rodríguez, Puebla, it is intended to know the trend of the legal constitution of companies according to article 19 of the Commercial Code, for this purpose, surveys were conducted on 87 economic units of this community, resulting in them that $58.63 \%$ are constituted and $69.44 \%$ of those that are not, state that they do not perform this task due to lack of time, on average $48.28 \%$ of the total respondents consider that the requirements for the constitution of their company are too many and difficult, $66.67 \%$ are natural persons and there is a lack of knowledge about their registration and where to do it according to their jurisdiction.
\end{abstract}

Keywords: Commercial Law, Public Registry of Commerce, MSMEs, Municipality of Tepexi de Rodríguez, Legal Constitution.

JEL: K22, K23, L51, 017, P37

Resumen Una de las obligaciones de todo comerciante es la inscripción de su negocio en el Registro Público de Comercio, esto garantiza conocimiento sobre las entidades económicas del país y fomenta el comercio formal. En Tepexi de Rodríguez, Puebla, se pretende conocer la tendencia de la constitución legal de empresas según el artículo 19 del Código de Comercio, para ello se realizaron encuestas a 87 unidades económicas de esta comunidad, dando como resultado de estas que el $58.63 \%$ están constituidas y el $69.44 \%$ de las que no lo están, manifiestan que no realizan dicha tarea por falta de tiempo, en promedio el $48.28 \%$ del total de los encuestados considera que son demasiados y difíciles los requisitos para la constitución de su empresa, el $66.67 \%$ son personas físicas y existe un desconocimiento sobre su registro y de donde realizarlo de acuerdo a su jurisdicción.

\section{Constitución legal y registro de las MiPymes de Tepexi de Rodríguez}


Palabras Clave: Derecho comercial, Registro Público de Comercio, MiPymes, Municipio de Tepexi de Rodríguez, Constitución legal.

\section{Introducción}

Son comerciantes aquellas personas que de manera diaria hacen de su vida diaria el comercio, esto significa que se ocupan en alguna de las actividades que el código de comercio de nuestro país considera actos mercantiles, mismos que se encuentran enlistados en el artículo 16 de esta ley; se consideran como tal: compra y venta de bienes muebles, inmuebles, acciones y demás practicas relacionadas con el abastecimiento, suministro, transporte, construcción, etc., por lo tanto, al realizar alguna de las actividades comerciales y generar ingresos económicos se tiene la obligación de contribuir al gasto público como lo marca el artículo 31 constitucional en su fracción cuarta.

Hoy en día las empresas establecidas en Tepexi de Rodríguez, Puebla que realizan actos de comercio en su mayoría son MIPYMES, toda vez que se caracterizan por contar con un número reducido de personal contemplando lo que considera Galindo (2001, pág. 44) una microempresa tiene de 0 a 10 trabajadores, la pequeña posee de 11 a 50 y la mediana abarca de 51 a 250. Este tipo de empresas desempeñan un papel muy importante en la economía y en el empleo a nivel regional, pues son las encargadas de aportar el poder adquisitivo y fuentes de empleo a los habitantes, lo que beneficia de manera positiva la economía de esta región.

En el último censo económico realizado por el INEGI en 2010 en la comunidad de Tepexi de Rodríguez, Puebla, existen ciento ochenta y siete unidades económicas de las cuales la mayoría son MIPYMES, de estas, solo ocho se encuentran registradas en el Sistema de Información Empresarial Mexicano (SIEM) cuyos datos son obtenidos por la Secretaria de Economía a través del Registro Público de Comercio. De lo anterior podemos determinar que solo unas cuantas empresas contribuyen al gasto público, el resto de estas entidades económicas influyen en la economía informal provocando la reducida recaudación de impuestos en el municipio como en el estado.

Es importante mencionar que desde una óptica legal una empresa constituida es vista de manera distinta ante sus socios o accionistas, clientes, proveedores y sociedad, ya que cuenta con la capacidad para adquirir derechos y obligaciones dentro de un marco jurídico, además de los

\section{Constitución legal y registro de las MiPymes de Tepexi de Rodríguez}


beneficios que son otorgados por el estado mexicano por ejemplo acreditar el importe de los estímulos fiscales a los que tengan derecho, conforme a lo estipulado en el artículo 25 del Código Fiscal de la Federación.

La finalidad de esta investigación es conocer cuántas MIPYMES ubicadas en la cabecera municipal de Tepexi de Rodríguez, Puebla, no han realizado la acción pertinente de constituirse legalmente, ya que el número de unidades económicas censadas por el INEGI no corresponde con las registradas por la Secretaria de Economía. De igual manera descubrir los motivos que orillan a los microempresarios de esta localidad a no realizar la constitución legal de sus empresas ante el Registro Público de la Propiedad y del Comercio y como resultado aumentar el comercio formal.

\title{
Marco teórico
}

\section{Antecedentes}

En las economías desarrolladas de la Organización para la Cooperación y el Desarrollo Económicos (OCDE) de 2004 las MIPYMES conforman una porción muy importante de la actividad económica, significando más del $90 \%$ de las empresas y generan más de la mitad del empleo que ofrece la actividad economía empresarial.

La participación de las MIPYMES en el Producto Interno Bruto (PIB) varía para estas economías, no obstante, se puede decir, que la participación en promedio se ubica en los alrededores del 50\% (OCDE, 2004). Participación que está en función del tipo de producción, por lo general es menor en la industria y mayor en el comercio y los servicios. El crecimiento de las MIPYMES, en estas economías, es el resultado de una amalgama de hechos, en donde se hace necesario destacar: los cambios estructurales generados por las fuerzas productivas universales, particularmente en la industria electrónica, que provocaron cambios en la industria de las comunicaciones y los servicios.

La Organización para la Cooperación y el Desarrollo Económicos (OCDE) cita a investigadores, como Fernando Villarán y Hernando de Soto que durante la década de los 80 , quienes difundieron la idea de las ventajas que ofrece las MIPYMES; otros autores como Peter Senge y Peter Drucker señalaban que todos podíamos ser empresarios tan solo identificando una

\author{
Pacheco, S., Escandón, R. \& Pérez, J.
}


oportunidad de negocio que nos permitiera en el largo plazo crecer y consolidar una empresa.

Cabe considerar que los principales problemas que afectan a la MIPYMES en México, están determinados por variables exógenas y endógenas al sistema económico, que pueden resumirse en: marco jurídico institucional; acceso y funcionamiento del mercado de factores productivos; introducción y ejercicio al mercado de bienes y servicios; gestión y cooperación empresarial y otros de carácter particular que están determinados por la región, la integración de familia, etc.

Dentro de esto un análisis del marco jurídico institucional de los negocios, revela que si bien han quedado superados, en teoría, aspectos de importancia como lo constituyen el marco regulatorios competitivo, como resultado de la nueva "Ley de Competencia"; la faltas de reformas estructurales que deben llevar a una modificación puntual del marco jurídico institucional siguen siendo una afectación directa en la búsqueda del incremento de su competitividad.

Como consecuencia de lo anterior el acceso al mercado de factores productivos muestra limitaciones, que se acentúan por el pobre nivel para lograr créditos bancarios, las estadísticas indican que la banca privada, para realizar préstamos debe contar con una gran cantidad de garantías, lo que resulta una situación difícil cuando las MIPYMES en México no cuentan, en general, con locales propios; además de los problemas de contabilidad que poseen estos tipos de empresas que las sitúan ante la banca como instituciones no sujetas a crédito. Por lo tanto, las MIPYMES tienen poca posibilidad de acceso a un mercado de trabajo donde exista la fuerza de trabajo calificada, que permita lograr los niveles de competitividad exigidos a nivel internacional y con la calidad requerida.

Dentro de este marco en México el Programa del presidente José López Portillo (1976-1982), pretendió la recuperación industrial por la vía de una mayor productividad de capital y de trabajo que trajeran consigo un gran desarrollo y fueran una fuente auto sostenida de empleo. Para ello, en 1979, se creó la Comisión Nacional de Fomento Industrial (CNFI) y la realización de foros de discusión y concertación que buscaran evitar la indefinición y la burocratización para impulsar el apoyo a la pequeña empresa. La mecánica a seguir consistió en otorgar a las MIPYMES, financiamiento y estímulos fiscales permitiendo a los grandes empresarios canalizar al exterior una

\section{Constitución legal y registro de las MiPymes de Tepexi de Rodríguez}


mayor proporción de su producción y operar mediante subcontratos con la pequeña y mediana industria. Posteriormente, el Plan Nacional de Desarrollo Nacional de 1982, implementó una política industrial que intentaba promover empleos a través de: el desarrollo ramos de alta productividad, la integración de la estrategia industrial para aprovechar los recursos naturales, la desconcentración territorial de la industria y la articulación de la gran empresa con la mediana y pequeña industria (Valdés Díaz de Villegas \& Sánchez Soto, 2012).

Hasta el presente no existe una ley que establezca efectivamente el marco para otorgar incentivos a las empresas. La Ley al Fomento a la Micro industria y a la Actividad Artesanal ha quedado como una declaración de buenas intenciones. La Ley Federal del Trabajo impide desarrollar mecanismos flexibles de contratación y remuneración al trabajo de las MIPYME. Por ello, las empresas pequeñas evitan contraer compromisos laborales pues los consideran muy riesgosos. La Ley del Seguro Social impone condiciones, requisitos difíciles de cumplir por una empresa pequeña, por lo que se necesita desarrollar un marco que les dé un trato especial. La Ley de Adquisiciones del Sector Público no da el mismo trato a las empresas pequeñas nacionales que a las empresas extranjeras; además, a pesar de la nueva clasificación de las MIPYME, es muy difícil que las microempresas puedan beneficiarse con este programa. Falta todavía claridad en normas oficiales y mecanismos para su aplicación en el mercado. (Saavedra García, Tapia Sánchez, \& Aguilar Anaya, 2013).

\section{Conceptos}

Definición de empresa. - Las empresas son organizaciones 0 unidades económicas productivas mediante las cuales sus propietarios, socios 0 accionistas deciden utilizar los factores de producción para obtener bienes y servicios que se ofrecen en el mercado a unos determinados compradores y/o consumidores (Olea Suarez \& Pacheco García, 2012).

Para el desarrollo de sus actividades cotidianas la empresa necesita disponer de técnicas y tecnologías que especifiquen el tipo de factores productivos que precisan y como se combinan. Así mismo, deben adoptar una organización y una forma jurídica pre-establecida que le permita

Pacheco, S., Escandón, R. \& Pérez, J. 
comercializar, realizar todo tipo de contratos, captar recursos financieros, entre muchas otras actividades.

Concepto de MIPYMES. - La palabra de "MIPYMES" está formada por las primeras letras de los conceptos micros, pequeñas y medianas empresas (Regalado H.R. 2007).

Aunque la definición puede variar según el país, dentro de este amplio concepto es posible englobar tanto a microemprendimiento (como podría ser la tienda del barrio), como firmas exportadoras y/o de alta tecnología, cuya facturación y número de empleados (en general de 1 a 150 empleados) está muy por debajo de las denominadas "grandes" empresas.

La clasificación que se maneja por el número de empleados establece que una microempresa tiene entre 1 y 10 empleados, la pequeña de 10 a 20; la mediana de 21 a 49; y la grande de 50 en adelante.

Empresario. - Se le considera empresario a la persona que aporta el capital y realiza en la mayoría de los casos y simultáneamente funciones propias de la dirección, planeación, organización de recursos y control. En muchos casos el origen de la empresa está en una idea innovadora sobre los procesos y productos, de forma que el empresario actúa como agente difusor del desarrollo económico.

Comercio. - Roberto Goldschmidt reafirma la falta de correspondencia entre las nociones económicas y jurídicas en relación al acto de comercio así: "El concepto del acto de comercio no se identifica con el del acto jurídico, sino que señala la actividad económica simple o compleja que se exterioriza en hechos y operaciones. Un acto de comercio puede componerse de varios actos jurídicos entre los cuales existe una vinculación desde el ángulo social y económico" (Morales Hernández, 1998).

Comercio informal. - El comercio informal será todo aquel que un individuo realice fuera de las estructuras formales del Estado, evadiendo el pago de impuestos y demás formalidades jurídicas. El ejemplo de comercio informal más común es aquel de los vendedores ambulantes, pero también aquel referido a negocios ilícitos, como la venta drogas prohibidas o el comercio de partes procedentes de robos o hurtos.

Comercio formal. - Por su parte, el comercio formal comprende aquel regulado por el Estado en el código de comercio, sujeto a la normativa legal vigente, y declarado con periodicidad ante los organismos fiscales competentes. La importancia del comercio formal es que este tiene la

\section{Constitución legal y registro de las MiPymes de Tepexi de Rodríguez}


capacidad de impulsar la economía de un país, dinamizarla, diversificarla, y producir riqueza para sus ciudadanos.

\section{Resultados}

El trabajo de investigación surge a partir de las clases de Taller de Investigación II en séptimo semestre y Derecho Empresarial de tercer semestre, ambas se cursaron durante el semestre Agosto-Diciembre 2017, asignaturas que se imparten en la Licenciatura en Administración del Instituto Tecnológico Superior de Tepexi de Rodríguez. Para esta indagación se aplicó un tipo de investigación cualitativa con un alcance exploratoriodescriptivo y de campo, a través de la cual se buscó conocer el interés y grado de disposición de los microempresarios de Tepexi de Rodríguez por cumplir los lineamientos establecidos por la ley para la constitución legal de sus empresas, así como las reglas que contribuyan al desarrollo de sus actividades con mayor eficiencia dentro del comercio formal.

Para la realización del estudio se tomó como referencia 87 MIPYMES ubicadas en la cabecera de dicho municipio. Se aplicaron técnicas de recolección de datos, tales como: investigación documental y encuesta. Para llevar a cabo esta tarea se elaboró un cuestionario con 10 preguntas, las cuáles versaban sobre el interés por tramitar la constitución legal, el registro de las empresas, así mismo sobre el conocimiento para realizar dicho trámite, estos reactivos se dividieron en dos tipos de acuerdo a la naturaleza de sus respuestas como lo muestra la tabla 1. Es importante señalar que la aplicación se realizó de manera directa a los microempresarios.

Finalmente, una vez recabados los datos arrojados por las encuestas se continuó a ordenarlos a través de gráficas que nos permitieron analizar y determinar los datos que nos exigen las interrogantes antes mencionadas.

Tabla 1. División de reactivos.

\begin{tabular}{ccc}
\hline No. De reactivo & \multicolumn{2}{c}{ Tipo de reactivo } \\
\cline { 2 - 2 } & Cerrada & $\begin{array}{c}\text { Opción } \\
\text { múltiple }\end{array}$ \\
\hline 1-.¿Está constituida su empresa o negocio? & $\mathrm{X}$ \\
\hline 2.-¿Cuenta con un representante legal? & $\mathrm{X}$ \\
\hline $\begin{array}{c}\text { 3.-¿Sabe los requisititos para la constitución legal de su } \\
\text { empresa o negocio? }\end{array}$ & $\mathrm{X}$ \\
\hline
\end{tabular}

Pacheco, S., Escandón, R. \& Pérez, J. 


\begin{tabular}{cc}
\hline $\begin{array}{c}\text { 4.-¿Conoce cuáles son las obligaciones comunes del } \\
\text { comerciante? }\end{array}$ & $\mathrm{X}$ \\
\hline $\begin{array}{c}\text { 5.-¿Considera que son demasiados los requisitos para la } \\
\text { constitución legal de una empresa? }\end{array}$ & $\mathrm{X}$ \\
\hline $\begin{array}{c}\text { 6.-¿Son difíciles de cumplir los requisitos para la } \\
\text { constitución legal de una empresa o negocio? }\end{array}$ & $\mathrm{X}$ \\
\hline 8.-¿El tipo de persona jurídica es moral? & $\mathrm{X}$ \\
\hline $\begin{array}{c}\text { 9.- ¿Conoce que posterior a la escritura o póliza } \\
\text { constitutiva debe realizar su inscripción al Registro } \\
\text { Público de Comercio? }\end{array}$ & $\mathrm{X}$ \\
\hline $\begin{array}{c}\text { 10.-¿ ¿Ubica dónde se encuentra el registro público de } \\
\text { Comercio en Tepexi de Rodríguez? }\end{array}$ & $\mathrm{X}$ \\
\hline 7.- Motivo por el cual no constituye su empresa o \\
negocio
\end{tabular}

Resumen de resultados

El no contar con un acta constitutiva no prohíbe la realización de una actividad comercial siempre y cuando este acto lo realice una persona física, sin embargo, es importante que los comerciantes realicen su constitución y registro en las dependencias correspondientes, paguen impuestos y sean sujetos a los beneficios que otorga el Estado. Debido a que la correcta creación de una empresa es trascendental ya que ayuda a evitar delitos asociados al comercio informal, por otro lado la identificación de esta situación no es de forma persecutoria sino preventiva que contribuye a estimular la regularización. 
Gráfica 1. Resultado a encuestados

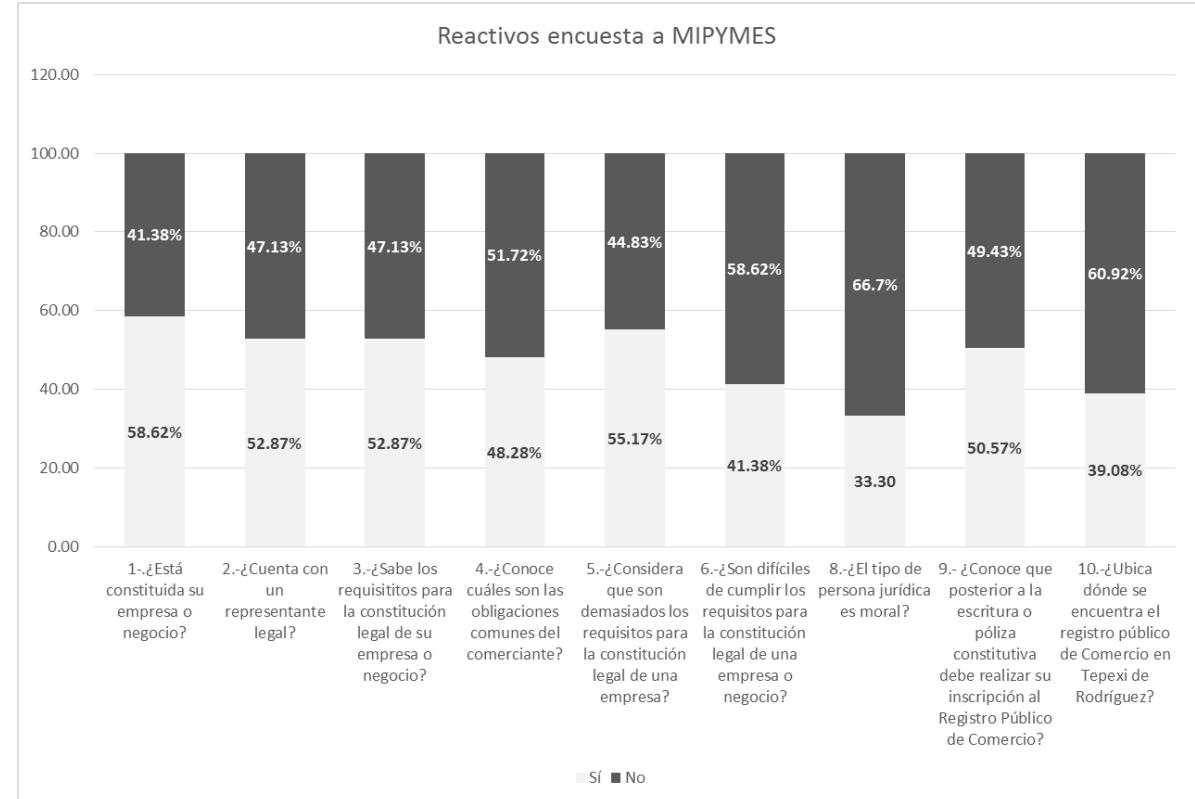

Fuente: Encuesta aplicada.

Gráfica 2. Motivos

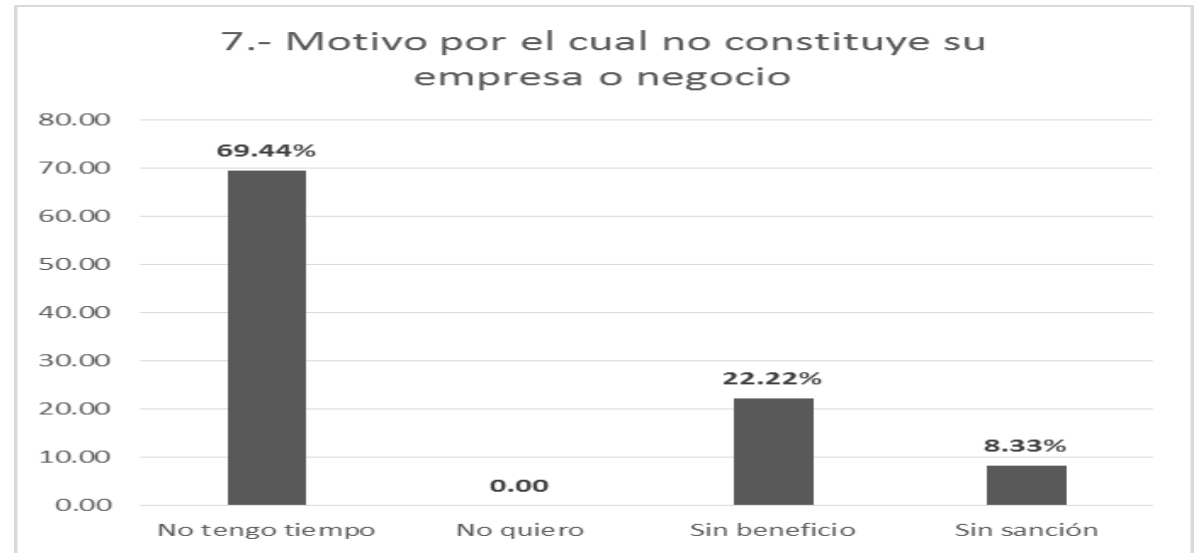

Fuente: Encuesta aplicada.

En la gráfica 1 se puede observar de los datos obtenidos, el $58.62 \%$ de los microempresarios a constituido su empresa, el $52.87 \%$ cuenta con un

Pacheco, S., Escandón, R. \& Pérez, J. 
representante legal, además el mismo porcentaje de encuestados conoce los requisitos para este trámite, nos percatamos que se tiene un escaso conocimiento del Código de Comercio mismo que se representa con un $51.72 \%$, el $55.17 \%$ considera que son demasiados requisitos para la constitución legal de su empresa o negocio, también se refleja que son difíciles de cumplir los requerimientos exigidos por la legislación esto lo confirma un $58.62 \%$. El $41.38 \%$ de encuestados no ha constituido su empresa, llama la atención que solo $50.57 \%$ de los encuestados conozca que posterior a la constitución de su negocio debe realizar su inscripción al Registro Público de Comercio, así mismo un $60.92 \%$ de estos desconoce donde se encuentra dicha dependencia u oficina en el municipio de Tepexi de Rodríguez y por último observamos que el $66.67 \%$ son personas físicas dejando el resto como personas morales.

Como se señala en la gráfica 2 el 69.44\% manifiesta que el principal motivo por el que no ha constituido su empresa es por falta de tiempo, mientras que el $22.22 \%$ considera no recibir beneficio alguno y un $8.33 \%$ opina no hacerse acreedor a ningún tipo de sanción.

\section{Discusión}

Este trabajo tiene una investigación en Tepexi de Rodríguez, Puebla existe el comercio informal toda vez que prevalece el desinterés por parte de los microempresarios para constituir legalmente sus empresas.

El número de MIPYMES constituidas es prácticamente la mitad de la muestra realizada y en similar proporción cuentan con un representante legal lo que nos indica que les preocupan los estatutos y leyes, sin embargo, la mayoría no conoce preceptos básicos de las obligaciones del comerciante además consideran son demasiados requisititos, de tal manera que son difíciles de cumplir.

Si bien, un gran número de los que no han realizado la constitución de su empresa manifiestan que es por falta de tiempo, es importante resaltar que casi la cuarta parte de estos considera que no recibe beneficio alguno y que el resto no lo hace porque presume no tener o recibir alguna sanción por parte del estado mexicano.

En mayor porcentaje los empresarios deciden registrarse como persona física en lugar de asociarse y constituir una empresa.

\section{Constitución legal y registro de las MiPymes de Tepexi de Rodríguez}


Finalmente, nos percatamos que en mayor medida los dueños de las MyPIMES desconocen donde se encuentran las oficinas del Registro Público de Comercio y que la mitad de ellos ignora que debe realizar este trámite.

\section{Recomendaciones}

Ante tal situación es necesario tomar medidas correctivas que propicien la concientización en los dueños de las MIPYMES en Tepexi de Rodríguez para constituir y registrar legalmente su empresa, para alcanzar mejores prácticas por lo que se sugieren las siguientes recomendaciones:

1) Brindar consultoría empresarial sobre derecho mercantil a los dueños de las MIPYMES de Tepexi de Rodríguez, Puebla por parte de los alumnos del Instituto Tecnológico Superior de Tepexi a través del Centro de Negocios de la institución donde se les dé a conocer cuáles son sus deberes y retribuciones en los aspectos legales sobre la constitución de sus empresas.

2) Analizar y elegir las estrategias para difundir la localización de las oficinas del Registro Público de la Propiedad en Tepexi de Rodríguez.

3) Diseñar y proponer un programa de concientización respecto del alta de los negocios ante instituciones gubernamentales federales, estatales y locales con la finalidad de regularizar el comercio informal en esta cabecera municipal.

\section{Referencias}

Constitución Política de los Estados Unidos Mexicanos. (05 de Febrero de 1917). Diario Oficial de la Federación.

Foddy, W. (1996). Constructing questions for interviews and questionnaires. Cambridge: Cambridge University Press.

Galindo, M. (2001). Fundamentos de Administración. México: Editorial Trillas.

INEGI. (11 de 05 de 2017). Instituto Nacional de Estadística y Geografía. Obtenido de http://www.inegi.org.mx/

Morales Hernández, A. (2007). Curso de Derecho Mercantil. Caracas: UCAB.

Olea Suarez, D. M., \& Pacheco García, R. (2012). Marketing la administración y la gerencia básica para las Mipymes colombianas. Bogotá, Colombia: Universidad Piloto de Colombia.

Regalado H, R. (2007). Las Mipymes en Latinoamerica. México: OLA.

Pacheco, S., Escandón, R. \& Pérez, J. 
Saavedra García, M. L., Tapia Sánchez, B., \& Aguilar Anaya, M. (2013). El impacto de las políticas públicas en la MIPYME mexicana. Ciencias Administrativas, 1(1), 1-19. Disponible en: https://revistas.unlp.edu.ar/CADM/article/download/659/549/

Valdés Díaz de Villegas, J. A., \& Sánchez Soto, G. A. (2012). Las MIPYMES en el contexto mundial: sus particularidades en México. Revista de Ciencias Sociales de la Universidad Iberoamericana, 2(14). 126-156. Disponible en: https://www.redalyc.org/pdf/2110/211026873005.pdf

Código de Comercio. (13 de Junio de 2014) Diario Oficial de la Federación. 